\title{
The role of attentional networks in voluntary task switching
}

\author{
Catherine M. Arrington and Melissa M. Yates \\ Lehigh University, Bethlehem, Pennsylvania
}

\begin{abstract}
Coordination of task choice and performance in multitask environments likely involves attentional processes. Subjects completed the Attention Network Test (ANT) and a voluntary task-switching procedure. Task choice, but not task performance, was correlated with the executive score from the ANT, with higher switch probabilities for subjects with more efficient executive control networks. Task performance was correlated with the alerting score, with larger response time switch costs for subjects with larger alerting scores. The dissociation of task choice and task performance measures in terms of the pattern of correlations with attentional networks suggests that these two measures may reflect different cognitive processes engaged in voluntary task switching.
\end{abstract}

Multitasking has become a buzzword of modern life, conjuring images from the CEO to the soccer mom. Anecdotally, individuals often endorse the idea that there are vast individual differences in how well people are able to multitask, with some individuals seemingly undeterred by the juggling act, whereas others suffer extreme performance failures in similar circumstances. What variation in basic cognitive operations may underlie these differences? In the present research, we examined how individual differences in three attentional networks (Posner \& Petersen, 1990) may be related to behavior in multitask environments. We measured variations in attentional function, using the Attention Network Test (ANT; Fan, McCandliss, Sommer, Raz, \& Posner, 2002), which provides measures of the alerting, orienting, and executive control networks, and correlated these measures with measures of task choice and performance in a voluntary task-switching paradigm (Arrington \& Logan, 2004).

\section{Task Switching}

Task-switching paradigms have become widespread in the study of cognitive control in multitask environments. Although a number of variants of the procedure exist, common features include the presentation of multivalent stimuli affording two or more simple categorization tasks and performance of the individual tasks in rapid succession. Key to the paradigm is the division of trial types into repetitions and switches. The standard finding is that performance on repetitions is faster and more accurate than switches. These performance differences, or switch costs, are used as measures of multitasking. A variety of interpretations of switch costs have been put forth in the literature, including active control mechanisms necessary to reconfigure the cognitive system for a new task when there is a switch, passive mechanisms such as priming of task performance when the task or elements of the task are repeated, and interference from previously active but no longer relevant task sets when the task is switched (see Monsell, 2003, for a concise review). The magnitude of switch costs has served as the dependent measure in a wide range of studies in which the factors influencing performance in multitask environments have been investigated. Furthermore, variation in switch costs has been examined in the context of studies in which individual differences in cognitive control processes have been investigated (Miyake et al., 2000; Yehene \& Meiran, 2007).

Voluntary task switching is a variant of the standard task-switching paradigms in which subjects are provided with no external cue or sequence that guides task choice (Arrington \& Logan, 2004). In this procedure, subjects are told to select one task on each trial, with only general instructions to perform the tasks equally often in a random order. Removing the external cue or sequence serves two primary functions. First, it requires that subjects exert control to guide task performance, since the external environment does not fully dictate the appropriate response, a concern raised about other task-switching procedures (Logan \& Bundesen, 2003; cf. Altmann, 2006). Second, since subjects select the task to perform on each trial, task choice becomes a second dependent measure of behavior in multitask environments. Choice can be considered in terms of both the task selected on a given trial and the task transition in terms of switch probability.

Previous research in which the voluntary task-switching procedure has been used has illuminated various factors that influence task choice. Subjects typically show a repetition bias, performing switches less often than would be expected if task choice were truly random. The repetition bias is influenced by the time available to prepare for the upcoming task, with subjects switching more as the

C.M.Arrington, kate.arrington@lehigh.edu 
response-stimulus interval (RSI) increases (Arrington \& Logan, 2005). This reduction in repetition bias is robust across both within- and between-subjects manipulations of RSI, suggesting that the control processes engaged in task choice are common across experimental manipulations (Liefooghe, Demanet, \& Vandierendonck, 2009). These results suggest that switching tasks requires time, making repetitions, under shorter time intervals, favored either strategically or by default. External factors also influence task choice, including stimulus repetition, which increases task repetitions (Mayr \& Bell, 2006), and stimulus availability, which increases the likelihood that a particular task will be performed (Arrington, 2008).

Researchers have considered individual differences in task performance measures in standard task-switching paradigms; however, little work has been done to examine individual differences in task choice in voluntary task switching. Mayr and Bell (2006) considered correlations between task performance and task choice measures across two pairs of tasks. They saw a strong correlation in switch probability across task domains, suggesting consistent individual differences in task choice. Furthermore, the variability in task choice was only weakly correlated with local response time (RT) switch costs, suggesting that choice and performance measures may capture different basic cognitive processes engaged during multitasking.

\section{Attentional Networks}

Intuitively, individual differences in multitasking behavior are likely to be linked to attentional differences; however, such intuitions raise the question of what aspects of attention are involved. Attention may be divided into processes that have both different functions in successful information processing and different neural bases. One influential theory of attention put forth by Posner and colleagues (Posner $\&$ Petersen, 1990; Posner \& Rothbart, 2007) carves attention into three separable networks: alerting, which maintains an alert state that is receptive to stimulus input and ready for responding; orienting, which involves selection of sensory input; and executive control, which monitors for and resolves conflict in responding or other aspects of cognitive processing. The ANT was designed to isolate the three attentional networks, using targeted contrasts between conditions within a single experimental paradigm (Fan et al., 2002). The procedure draws from two long-standing paradigms used in empirical examinations of attention: the cuing task and the flanker task. Each trial begins with a cue (except for the no-cue condition) that indicates that a target is about to appear (central-cue and double-cue conditions) and where it will appear (spatial-cue condition). The target display appearing above or below fixation includes a central target arrow surrounded by flanker arrows that are either compatible with the target (pointing in the same direction) or incompatible (pointing in the opposite direction). The alerting network is indexed through contrasting RTs from the no-cue and double-cue conditions. The orienting network is indexed through contrasting RTs from the central-cue and spatial-cue conditions. The executive network is indexed through contrasting RTs from the compatible and incompatible flanker conditions.
The measures of the alerting, orienting, and executive networks have been shown to be reliable measures of individual differences in the efficiency of the different attentional networks (Fan et al., 2002; cf. Redick \& Engle, 2006). The measures show only small correlations among networks, suggesting largely independent functioning of the networks (Fan et al., 2002). This independence is also supported by neuroimaging studies that delineate separate brain regions underlying the networks (Fan, McCandliss, Fossella, Flombaum, \& Posner, 2005). Since its development, the ANT has been widely used to examine variation in the different attentional networks associated with stages of development, training experience, behavioral and neurological disorders, and genetic variations (see Posner \& Rothbart, 2007, for a comprehensive review).

The present study addresses the question of whether individual differences in task choice and performance in the voluntary task-switching procedure are linked to differences in efficiency of the three attentional networks. This individual differences approach may provide leverage in understanding the cognitive processes underlying behavior in multitask environments.

\section{METHOD}

\section{Subjects}

Sixty-four undergraduate subjects completed both tests. Data from 7 subjects were removed from the final analysis due to accuracy below $90 \%$ on either the ANT or the voluntary task-switching procedure. All the subjects reported normal or corrected-to-normal vision.

\section{Overall Procedure}

All stimulus presentation and data collection were conducted on a Dell Dimension with a 17 -in. CRT monitor. Both protocols were run using the E-Prime software package. The subjects were seated in a dimly lit room, and viewing distance was unconstrained. The order of the two procedures was counterbalanced across subjects.

\section{Voluntary Task Switching}

The subjects performed even/odd judgments on digits and consonant/vowel judgments on letters. The stimuli were the numbers 2-9 and the letters A, B, C, E, I, L, U, and W, presented in black on a light gray background. The stimuli were $7 \times 5 \mathrm{~mm}$ and appeared $20 \mathrm{~mm}$ from fixation along the diagonals. Each trial began with the onset of a fixation screen containing a cross in the center of the display and four pound signs (\#) marking the possible target locations. The fixation display remained on the screen for either 200 or $1,000 \mathrm{msec}$, followed by the onset of a digit and a letter at two randomly selected locations. The targets remained on the screen until a response occurred. Responses were made with the index and middle fingers of each hand, with the hand-to-task mapping counterbalanced across subjects. The subjects performed 16 practice trials for each task and then received standard voluntary task-switching instructions to perform the tasks equally often in a random sequence, modeled on published instructions (Arrington \& Logan, 2004). The subjects performed one practice block of task-switching trials and then performed four blocks of 96 trials. $^{1}$

\section{ANT}

The version of the ANT used followed the methods and procedures previously described by Fan et al. (2002) and made available for download by that research group. The subjects indicated whether a centrally located arrow was pointing to the left or right by making a buttonpress response on a two-button mouse held so that the thumbs rested on the two response keys. Two independent variables 
A

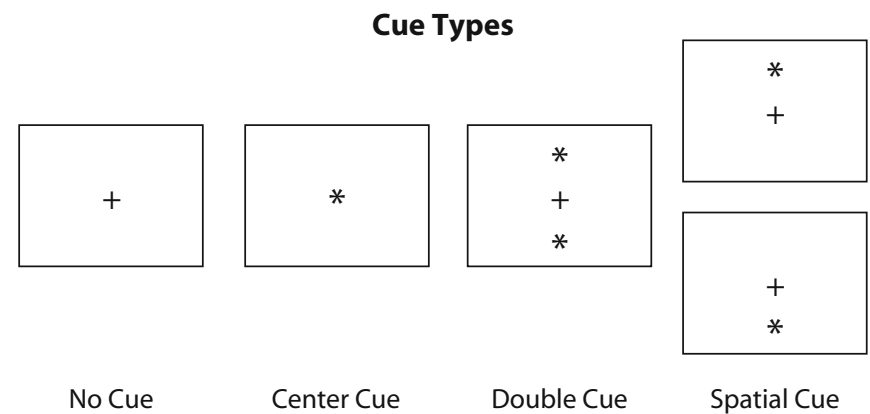

B

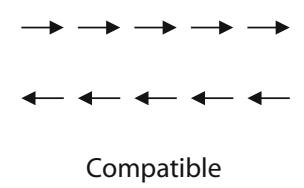

C

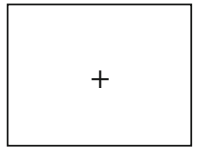

$400-1,600$ msec
$100 \mathrm{msec}$

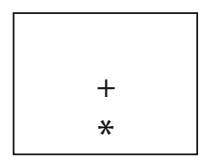

\section{Flanker Types}

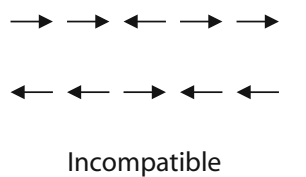

Trial Line

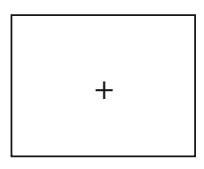

$400 \mathrm{msec}$

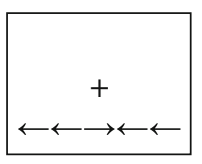

Response Time

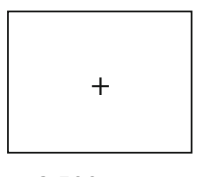

$3,500 \mathrm{msec}$

Figure 1. Examples of the design and procedure from the Attention Network Test. (A) The four cue types. (B) The three flanker types. (C) An example of the trial line showing the order and timing of events.

were manipulated: cue type and flanker type. Schematics of the different cue and flanker conditions, along with an example trial line, are shown in Figure 1. The subjects completed 24 practice trials with accuracy feedback, followed by three blocks of 96 trials without accuracy feedback.

\section{RESULTS}

\section{Voluntary Task Switching}

Trials were sorted into task and transition conditions on the basis of the responses performed on trial $n$ and trial $n-1$. Errors, trials following errors, and the first trial of each block were excluded from choice analyses. RT analyses further excluded trials on which the RT was less than $200 \mathrm{msec}$ or greater than $3,000 \mathrm{msec}$. Summary switch probability, RT, and response accuracy data are provided in Table 1. Responses were faster for repetitions than for switches. Switch costs were $256 \mathrm{msec}$ at the short RSI and $170 \mathrm{msec}$ at the long RSI, thus decreasing as RSI increased. A 2 (task transition: repetition or switch) $\times 2$ (RSI: 200 or $1,000 \mathrm{msec})$ repeated measures ANOVA showed significant main effects of task transition $[F(1,56)=194.7, p<$ $.05]$ and $\operatorname{RSI}[F(1,56)=4.42, p<.05]$, as well as the interaction of these two factors $[F(1,56)=48.1, p<.05]$. Response accuracy also showed more accurate performance on repetitions than on switches $[F(1,56)=9.5, p<.05]$, with the magnitude of the difference interacting with RSI $[F(1,56)=5.4, p<.05]$.
The task choice data also showed the expected pattern of effects, with subjects switching less frequently than would be expected from a random task sequence. The switch probability increased significantly as the RSI increased $[t(56)=10.3, p<.05]$. The effect of stimulus repetition was also considered. The subjects were no less likely to switch tasks if the stimulus on which the task was performed on trial $n-1$ was repeated on trial $n(.396)$ than if the stimulus was switched $(.388)[t(56)<1]$. This finding fails to replicate Mayr and Bell's (2006) stimulus repetition effect but may result from having separate stimuli for each task.

Table 1

Means and Standard Deviations for Switch Probability, Response Time, and Response Accuracy Measures in the Voluntary Task-Switching Procedure

\begin{tabular}{|c|c|c|c|c|}
\hline \multirow[b]{3}{*}{ Measure } & \multicolumn{4}{|c|}{ Response-Stimulus Interval } \\
\hline & \multicolumn{2}{|c|}{$200 \mathrm{msec}$} & \multicolumn{2}{|c|}{$1,000 \mathrm{msec}$} \\
\hline & $M$ & $S D$ & $M$ & $S D$ \\
\hline Switch Probability & .336 & .117 & .427 & .116 \\
\hline \multicolumn{5}{|l|}{ Response Time } \\
\hline Repetition & 936 & 118 & 960 & 119 \\
\hline Switch & 1,192 & 190 & 1,130 & 177 \\
\hline \multicolumn{5}{|l|}{ Response Accuracy } \\
\hline Repetition & 97.2 & 1.9 & 96.5 & 2.5 \\
\hline Switch & 95.4 & 3.9 & 96.0 & 3.7 \\
\hline
\end{tabular}


Table 2

Means and Standard Deviations for Response Time and Accuracy Measures Based on Cue Type and Flanker Type in the Attention Network Test

\begin{tabular}{|c|c|c|c|c|c|c|c|c|}
\hline \multirow[b]{3}{*}{ Measure } & \multicolumn{8}{|c|}{ Cue Type } \\
\hline & \multicolumn{2}{|c|}{ No } & \multicolumn{2}{|c|}{ Center } & \multicolumn{2}{|c|}{ Double } & \multicolumn{2}{|c|}{ Spatial } \\
\hline & $M$ & $S D$ & $M$ & $\overline{S D}$ & $M$ & $\overline{S D}$ & $M$ & $S D$ \\
\hline \multicolumn{9}{|l|}{ Response Time } \\
\hline Congruent & 540 & 72 & 502 & 71 & 489 & 61 & 460 & 71 \\
\hline Incongruent & 647 & 99 & 628 & 93 & 610 & 86 & 563 & 93 \\
\hline Neutral & 528 & 64 & 494 & 64 & 480 & 61 & 455 & 64 \\
\hline \multicolumn{9}{|c|}{ Response Accuracy } \\
\hline Congruent & 99.4 & 1.5 & 99.2 & 2.0 & 99.4 & 1.7 & 99.5 & 1.4 \\
\hline Incongruent & 94.7 & 5.3 & 92.3 & 7.8 & 92.6 & 7.6 & 96.9 & 4.2 \\
\hline Neutral & 99.1 & 1.9 & 98.9 & 2.0 & 98.9 & 2.7 & 99.5 & 1.4 \\
\hline
\end{tabular}

\section{ANT}

Mean RTs for each condition were calculated after removing error trials and trials on which the RT was less than $200 \mathrm{msec}$ or greater than 1,700 msec (see Table 2 for summary statistics). The pattern of results replicated those seen in previous experiments using the ANT (Fan et al., 2002). Measures of the efficiency of the three attentional networks were calculated for each individual from planned contrasts between conditions. The alerting network was assessed by subtracting the double-cue from the no-cue condition, thus looking at the effect of forewarning of the upcoming target. The mean $(S D)$ of the alerting score was $46 \mathrm{msec}$ (24). The orienting network was measured by subtracting the single-cue from the center-cue condition, thus capturing the effect of knowing where an upcoming target would appear. The mean $(S D)$ of the orienting score was $47 \mathrm{msec}$ (25). The executive network was measured by subtracting the compatible from the incompatible flanker condition, which provides a measure of how well conflict is managed. The mean $(S D)$ of the executive score was $114 \mathrm{msec}$ (38). Correlations among the three measures (shown in Table 3 ) were all nonsignificant, reinforcing the independence of the three networks.

\section{Correlation Analyses}

The primary question of interest in the present study was whether the efficiency of the three attentional networks would correlate with behavior in the voluntary taskswitching procedure. To address this question, we corre- lated ANT scores with both task choice (switch probability) and task performance (switch cost) data. Table 3 provides the results of these analyses. ${ }^{2}$ The alerting score correlated with switch costs, so that those individuals who had larger alerting scores also showed greater switch costs. The orienting score did not correlate significantly with either measure from the voluntary task-switching procedure. The executive score correlated negatively with switch probability. The subjects with smaller executive scores were better able to resolve the conflict associated with incompatible flankers. Thus, the negative correlation suggests that those subjects able to resolve conflict more efficiently were more likely to switch tasks in the voluntary task-switching procedure. The correlations did not differ significantly as a function of RSI, for both the correlations between the alerting score and task performance and those between the executive score and task choice. The lack of an effect of RSI indicates that the relationships were consistent across varying levels of preparation time. Correlations between the three ANT measures and the response accuracy measures from the voluntary task-switching paradigm yielded only one significant correlation between the executive score and accuracy on the switch trials at the long RSI $(r=.29, p<.05)$.

Finally, to fully examine the nature of individual differences in voluntary task-switching behavior, we correlated task performance and task choice measures within the procedure. As can be seen in Table 3, the switch probability showed no significant correlation with switch costs. This result seems in line with the findings of Mayr and Bell (2006), who showed weaker correlations of switch probability with local switch costs (as measured here) than with global switch costs (the difference between repetition trials and single task trials). Lastly, the switch probability correlated negatively with the effect of stimulus repetition $(r=-.37, p<.05)$. This correlation indicates that those individuals who chose to switch more frequently were less likely to be influenced by the stimulus repetition (Mayr \& Bell, 2006).

\section{DISCUSSION}

Voluntary behavior in multitask environments is flexible, thus allowing for individual variation in the control of the cognitive operations that are deployed for coordina-

Table 3

Correlations Between the Three Components of the Attention Network Test and Task Choice and Performance Measures From the Voluntary Task-Switching Procedure

\begin{tabular}{|c|c|c|c|c|c|c|c|c|}
\hline Measure & 1 & 2 & 3 & 4 & 5 & 6 & 7 & 8 \\
\hline \multicolumn{9}{|l|}{ ANT Scores } \\
\hline 1. Alerting & - & & & & & & & \\
\hline 2. Orienting & .028 & - & & & & & & \\
\hline 3. Executive control & .171 & -.045 & - & & & & & \\
\hline \multicolumn{9}{|l|}{ Voluntary Task Switching } \\
\hline 4. Overall response time & .024 & -.088 & .035 & - & & & & \\
\hline 5. Switch costs 200-msec RSI & $.260^{\dagger}$ & .022 & .016 & $.485^{*}$ & - & & & \\
\hline 6. Switch costs $1,000-\mathrm{msec}$ RSI & $.309^{*}$ & .068 & .042 & $.422^{*}$ & $.729^{*}$ & - & & \\
\hline 7. Switch probability $200-\mathrm{msec}$ RSI & -.171 & -.136 & $-.273^{*}$ & $.349^{*}$ & -.118 & -.128 & - & \\
\hline 8. Switch probability $1,000-\mathrm{msec}$ RSI & -.188 & -.041 & $-.368^{*}$ & .211 & -.154 & -.223 & $.837^{*}$ & - \\
\hline
\end{tabular}

Note-RSI, response-stimulus interval. ${ }^{*}$ Significance at the $p<.05$ level. †Significance level for this correlation was $p=.051$. 
tion of individual task performance and switching between tasks. We investigated the relationship among task choice and performance in a voluntary task-switching procedure (Arrington \& Logan, 2004) and the three attentional networks indexed by the ANT (Fan et al., 2002): alerting, orienting, and executive control. The alerting and executive networks correlated with task performance and task choice measures of multitask behavior, respectively.

Task-switching procedures are often assumed to measure executive control processes (Monsell, 2003). As such, it was predicted that the executive score of the ANT would correlate with behavior in the voluntary task-switching procedure. The correlation with the executive score occurred only for the task choice, but not for task performance measures. In the present paradigm, two stimuli appear on every trial affording both possible tasks. Since both tasks are available, responses for both tasks may become activated on each trial, just as responses for both the target and incompatible flankers may be activated in the ANT. The resolution of that conflict is necessary for subjects to successfully complete a single task. Those subjects with an efficient executive network may be better able to resolve the conflict between competing tasks even when switching between the tasks, which results in reconfiguration of task set and weaker instantiation of the current task set. Selection of a task is proposed to involve inhibition of competing task sets (Lien \& Ruthruff, 2008; Mayr \& Bell, 2006), which may be a more pronounced strategy for individuals with more efficient executive networks. Evidence from neuroimaging studies suggests overlap in regions of the cingulate cortex associated with the executive network (Fan et al., 2005) and voluntary selection of task sets (Forstmann, Brass, Koch, \& von Cramon, 2006).

The lack of a correlation between switch costs and the executive score suggests that this performance measure may not be closely connected to executive control. One consideration in the interpretation of this relationship is the limited scope of the executive component of the ANT. On the basis of a comparison between compatible and incompatible flanker trials, the score represents a narrow slice of executive control-namely, conflict resolution. Interestingly, Yehene and Meiran (2007) found weak correlations for congruency effects across task-switching paradigms, suggesting that the RTs associated with conflict resolution may be specific to the paradigm in which the conflict occurs. Clearly, other processes not captured by this contrast fall into the realm of executive control (Redick \& Engle, 2006). This inherent limitation raises the possibility that other measures of executive control may share variance with task-switching measures. However, other studies have failed to show such a correlation between RT switch costs and variation in working memory capacity (Kane, Conway, Hambrick, \& Engle, 2007; Unsworth \& Engle, 2008). These findings raise the question of whether switch costs reflect executive control processes engaged on switch trials or some other mechanism, such as passive interference or priming from previous task sets (Allport, Styles, \& Hsieh, 1994; Logan \& Bundesen, 2003).

In contrast to the executive score, the alerting score was correlated with switch costs, but not with switch probabil- ity. ${ }^{3}$ Large alerting scores tend to arise from disproportionately slow responding in the no-cue condition, rather than faster responding in the double-cue condition, suggesting that these subjects are not able to maintain a vigilant state in the absence of external support. Likewise, the larger switch costs result from slowing on switch trials, rather than speeding on repetition trials. Subjects less able or motivated to maintain alertness throughout the transition between trials may delay task set reconfiguration on switch trials until the external target appears. Closer examination of the present data showed that switching tasks was particularly slowed for the 1,000 -msec RSI for individuals with large alerting scores, relative to those with small alerting scores, suggesting that these subjects did not benefit as much from the longer preparation interval. This result would be expected if they were not maintaining alertness during the time between trials.

No correlations were found between the orienting score and the voluntary task-switching measures. This result may be due to the relatively limited nature of the stimulus input in the present procedure, rather than any true dissociation between orienting efficiency and multitask behavior. The sparse display elements were presented in a small number of central, fixed locations; thus, little demand for spatial orienting was placed on the subjects. Multitasking in richer or dynamic sensory environments requiring greater orienting may show correlations between orienting efficiency and multitask behavior.

Within the present data set, several pieces of evidence point toward the conclusion that task choice (switch probability) and task performance (switch costs) measures result from at least partially separate cognitive processes. Within the voluntary task-switching procedure, the switch probability and switch costs are, at most, weakly correlated. Furthermore, the correlations between the attentional networks and the voluntary task-switching measures also indicate a separation between the two measures. Task choice and task performance may engage separate stages of processing. It may be useful to consider this separation within Logan and Gordon's (2001) model of executive control, which proposes two levels of task control: a propositional level held in working memory and a parameter level transmitted to the subordinate systems that carry out attention and response selection processes. Task choice may involve forming an intention to perform a given task at the propositional level. As has been described by Arrington and Logan (2005), under the instructions to perform tasks in a random order, this selection process may involve holding in working memory a series of recently performed tasks and choosing a task that makes that sequence match some internal representation of randomness. Alternatively, under conditions that reduce the ability to perform this controlled selection process, such as time pressure or working memory load, individuals may use a simple heuristic of selecting the task most available to working memory. Once the intention to perform a task is formed, configuration of the cognitive system to perform the selected task is implemented by transmission of the task parameters to the subordinate systems. Switch costs may result from the extra time needed to transmit 
parameters on switch trials or weaker instantiation of the task set (Logan \& Gordon, 2001). Further empirical work will be necessary to clarify the nature of these separate but related aspects of voluntary behavior in multitasking.

\section{AUTHOR NOTE}

The authors thank Iring Koch, André Vandierendonck, and an anonymous reviewer for thoughtful comments on an earlier draft of the manuscript. Correspondence should be addressed to C. M. Arrington, Department of Psychology, Lehigh University, 17 Memorial Drive East, Bethlehem, PA 18015 (e-mail: kate.arrington@lehigh.edu).

\section{REFERENCES}

Allport, A., Styles, E. A., \& Hsieh, S. (1994). Shifting intentional set: Exploring the dynamic control of tasks. In C. Umiltà \& M. Moscovitch (Eds.), Attention and performance XV: Conscious and nonconscious information processing (pp. 421-452). Cambridge, MA: MIT Press.

Altmann, E. M. (2006). Task switching is not cue switching. Psychonomic Bulletin \& Review, 13, 1016-1022.

Arrington, C. M. (2008). The effect of stimulus availability on task choice in voluntary task switching. Memory \& Cognition, 36, 991997. doi:10.3758/MC.36.5.991

Arrington, C. M., \& Logan, G. D. (2004). The cost of a voluntary task switch. Psychological Science, 15, 610-615. doi:10.1111/j.0956 -7976.2004.00728.x

ArRington, C. M., \& Logan, G. D. (2005). Voluntary task switching: Chasing the elusive homunculus. Journal of Experimental Psychology: Learning, Memory, \& Cognition, 31, 683-702. doi:10.1037/0278 $-7393.31 .4 .683$

Fan, J., McCandliss, B. D., Fossella, J., Flombaum, J. I., \& Posner, M. I. (2005). The activation of attentional networks. NeuroImage, 26, 471-479. doi:10.1016/j.neuroimage.2005.02.004

Fan, J., McCandliss, B. D., Sommer, T., Raz, A., \& Posner, M. I. (2002). Testing the efficiency and independence of attentional networks. Journal of Cognitive Neuroscience, 14, 340-347. doi:10.1162/089892902317361886

Forstmann, B. U., Brass, M., Koch, I., \& von Cramon, D. Y. (2006). Voluntary selection of task sets revealed by functional magnetic resonance imaging. Journal of Cognitive Neuroscience, 18, 388-398. doi:10.1162/089892906775990589

Kane, M. J., Conway, A. R. A., Hambrick, D. Z., \& Engle, R. W. (2007). Variation in working memory capacity as a variation in executive attention and control. In A. R. A. Conway, C. Jarrold, M. J. Kane, A. Miyake, \& J. N. Towse (Eds.), Variation in working memory (pp. 21-48). New York: Oxford University Press.

Liefooghe, B., Demanet, J., \& Vandierendonck, A. (2009). Is advance reconfiguration in voluntary task switching affected by the design employed? Quarterly Journal of Experimental Psychology, 62, 850-857.

Lien, M.-C., \& RuthrufF, E. (2008). Inhibition of task set: Converging evidence from task choice in the voluntary task-switching paradigm. Psychonomic Bulletin \& Review, 15, 1111-1116. doi:10.3758/ PBR.15.6.1111

Logan, G. D., \& Bundesen, C. (2003). Clever homunculus: Is there an endogenous act of control in the explicit task cuing procedure? Journal of Experimental Psychology: Human Perception \& Performance, 29, 575-599. doi:10.1037/0096-1523.29.3.575

Logan, G. D., \& GoRdon, R. D. (2001). Executive control of visual attention in dual-task situations. Psychological Review, 108, 393-434. doi:10.1037/0033-295X.108.2.393

MAYr, U., \& BeLl, T. (2006). On how to be unpredictable: Evidence from the voluntary task-switching paradigm. Psychological Science, 17, 774-780. doi:10.1111/j.1467-9280.2006.01781.x

Miyake, A., Friedman, N. P., Emerson, M. J., Witzki, A. H., HowERTER, A., \& Wager, T. D. (2000). The unity and diversity of executive functions and their contributions to complex "frontal lobe" tasks: A latent variable analysis. Cognitive Psychology, 41, 49-100. doi:10.1006/cogp.1999.0734

Monsell, S. (2003). Task switching. Trends in Cognitive Sciences, 7, 134-140. doi:10.1016/S1364-6613(03)00170-0

Posner, M. I., \& Petersen, S. E. (1990). The attention system of the human brain. Annual Review of Neuroscience, 13, 25-42. doi:10.1146/ annurev.ne.13.030190.000325

Posner, M. I., \& Rothbart, M. K. (2007). Research on attention networks as a model for the integration of psychological science. Annual Review of Psychology, 58, 1-23. doi:10.1146/annurev .psych.58.110405.085516

REDICK, T. S., \& ENGLE, R. W. (2006). Working memory capacity and Attention Network Test performance. Applied Cognitive Psychology, 20, 713-721. doi:10.1002/acp.1224

Unsworth, N., \& Engle, R. W. (2008). Speed and accuracy of accessing information in working memory: An individual differences investigation of focus switching. Journal of Experimental Psychology: Learning, Memory, \& Cognition, 34, 616-630. doi:10.1037/0278 $-7393.34 .3 .616$

Yehene, E., \& Meiran, N. (2007). Is there a general task switching ability? Acta Psychologica, 126, 169-195. doi:10.1016/j.actpsy.2006 .11 .007

\section{NOTES}

1. Within the testing session, all the subjects followed the performance of the described voluntary task-switching protocol with a second version. That version included a manipulation of the stimulus onset asynchrony to allow for consideration of the influence of external stimulus availability on task choice (Arrington, 2008). The data from this manipulation are not considered here; however, since this condition was always run after the current voluntary task-switching condition, it should not have influenced the results.

2. When interpreting these correlations, it is important to consider the reliability of the measures being correlated, particularly in the case of the ANT scores and switch cost measures, since these reflect difference scores. Reliability of the three scores from the ANT was assessed by calculating the correlations between scores calculated from the even versus odd trials of each trial type. Correlations were significant for all three scores: alerting (.30), orienting (.51), and executive (.68). A similar splithalf procedure used for the switch cost measures showed significant correlations for the short RSI (.62) and the long RSI (.50). Given the sequential nature of the switch probability measure, splitting the data on the basis of even versus odd trials was not warranted. Instead, switch probabilities from each of the four blocks of data were used to calculate Cronbach's alpha, which was .87 for the short RSI and .89 for the long RSI.

3. Although the correlations between the alerting score and switch probability were not significant, further analyses showed that they also did not differ significantly from the correlations between the executive score and switch probability.

(Manuscript received September 27, 2008; revision accepted for publication March 29, 2009.) 